\title{
PAPER
}

\section{A prospective study of secondary degeneration following subcortical infarction using diffusion tensor imaging}

\author{
Zhijian Liang, Jinsheng Zeng, Sirun Liu, Xueying Ling, Anding Xu, \\ Jian Yu, Li Ling
}

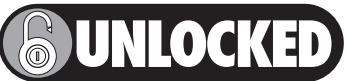

This paper is freely available online under the BMJ Journals unlocked scheme, see http://jnnp.com/info/unlocked.dtl

See Editorial Commentary, p 554

J Neurol Neurosurg Psychiatry 2007;78:581-586. doi: 10.1136/jnnp.2006.099077

See end of article for authors' affiliations

......................

Correspondence to: Professor Jinsheng Zeng, Department of Neurology and Stroke Centre, The First Affiliated Hospital, Sun YatSen University, No 58 Zhongshan Road II, Guangzhou, 510080, PR China;

zengjs@

pub.guangzhou.gd.cn

Received 1 June 2006

Revised 4 January 2007

Accepted 5 January 2007

Published Online First

23 January 2007
Background: Secondary degeneration of the pyramidal tract distal to the primary lesion after a stroke has been detected by some studies using diffusion tensor imaging (DTI) but its potential clinical significance and the degeneration of the fibre tract proximal to the primary lesion have received little attention.

Methods: Twelve patients underwent DTI on the 1st, 4th and 12th week following a subcortical infarct involving the posterior limb of the internal capsule, and 12 age and sex matched controls underwent DTI once. The DTI parameters mean diffusivity and fractional anisotropy (FA), and the clinical scores before DTI examination, including the National Institutes of Health Stroke Scale (NIHSS), the Fugl-Meyer (FM) scale and the Barthel index (BI), were assessed. The relations between the per cent changes in DTI parameters and clinical scores were analysed.

Results: From the 1st to the 12th week after stroke onset, FA values decreased ( $p<0.01$, respectively) in the fibre tract above and below the internal capsule, and the NIHSS decreased $(p<0.01)$ but the FM scale and BI increased ( $p<0.01$, respectively) progressively. The per cent reductions in FA value in the fibre tract above and below the internal capsule were negatively correlated with the per cent changes in NIHSS and FM scale $(\mathrm{p}<0.05$, respectively).

Conclusions: Secondary degeneration of the fibre tract proximal and distal to a primary lesion can be detected by DTI clearly and quantitatively and deteriorates with time progressively, which may hamper functional recovery after a subcortical cerebral infarct.
A nimal experiments and post-mortem examinations have demonstrated that a focal cerebral infarct can cause secondary degeneration in fibre pathways remote from the primary lesion. Delayed disintegration of such a fibre tract is considered to be Wallerian degeneration (WD), defined as anterograde degeneration of a nerve tract distal to an injury. ${ }^{1-5}$ Conventional MRI can detect the ipsilateral cerebral peduncle atrophy during the chronic stage of a focal cortical infarct ${ }^{6-8}$ but cannot reveal the delayed degeneration in the pyramidal tract on other regions clearly or quantitatively. Diffusion tensor imaging (DTI), which uses diffusion sensitive gradients applied in at least six non-collinear directions, can determine the diffusivity of every voxel and fully depict tissue diffusion characteristics. DTI has been used to detect and quantify the secondary degeneration in the fibre tract in vivo. ${ }^{9} 10$

Thomalla et $a l^{11}$ reported that secondary degeneration revealed by DTI occurred in the pyramidal tract distal to the primary lesion from the acute ( 5 days from onset) to the chronic (288 days) stage in two patients with ischaemic stroke. In some cross sectional studies, fractional anisotropy (FA) values were found to be generally reduced along the pyramidal tract on the infarct side distal to the primary lesion. ${ }^{9}{ }^{12}{ }^{13}$ After middle cerebral artery territory infarction, lower FA values in the cerebral peduncle were associated with a greater neurological deficit acutely, with worse outcomes 3 months later. ${ }^{13}$ However, to date, no prospective, controlled, contrast enhanced studies have monitored secondary degeneration, and the potential clinical significance of the degeneration distal to the primary lesion has not been confirmed. In addition, only one case report revealed that a pontine infarct can cause retrograde degeneration in the fibre pathway proximal to the primary lesion. ${ }^{14}$ However, there has been little research on the retrograde degeneration of the fibre tract after subcortical infarction, and its impact on the patient's outcome is not well understood.

In this study, DTI was used to prospectively quantify and monitor changes in diffusivity in the fibre pathway both proximal and distal to a recent subcortical infarct in 12 patients. The relation between the per cent changes in DTI parameters and clinical scores were analysed.

\section{MATERIAL AND METHODS \\ Subjects}

We selected 12 consecutive patients within 7 days of stroke onset who had unilateral subcortical cerebral infarct involving the posterior limb of the internal capsule. Patients were excluded if they had other signal abnormalities on T1 and T2 weighted images and fluid attenuated inversion recovery (FLAIR) images on magnetic resonance. No patient had a history of brain or spinal cord disease. Following a predefined protocol, all patients had DTI in the first week (Wl, 5 (2) days), at the end of the fourth week (W4, 28 (2) days) and at the end of the 12th week (W12, 88 (2) days).

Patient demographics and vascular risk factors were obtained. All patients had a detailed neurological examination $2 \mathrm{~h}$ before the magnetic resonance examination, including an evaluation of neurological deficits using the National Institutes of Health Stroke Scale (NIHSS), motor deficits using the FuglMeyer (FM) scale and a life independence assessment using the

Abbreviations: $\mathrm{BI}$, Barthel Index; DTI, diffusion tensor imaging; FA, fractional anisotropy; FLAIR, fluid attenuated inversion recovery; FM, FuglMeyer; MD, mean diffusivity; NIHSS, National Institutes of Health Stroke Scale; ROI, region of interest; WD, Wallerian degeneration 
Table 1 Patient demographics and clinical data

\begin{tabular}{|c|c|c|c|c|c|c|}
\hline \multirow[b]{2}{*}{$\begin{array}{l}\text { Patient } \\
\text { No }\end{array}$} & \multirow[b]{2}{*}{$\begin{array}{l}\text { Age } \\
\text { (y) }\end{array}$} & \multirow[b]{2}{*}{ Sex } & & \multicolumn{3}{|c|}{ Infarct on FLAIR images } \\
\hline & & & & Side & $\begin{array}{l}\text { Volume of lesion } \\
\left(\mathrm{mm}^{3}\right)\end{array}$ & Site of lesion \\
\hline 1 & 46 & M & $\begin{array}{l}\text { Hypertension, hypercholesterolaemia, } \\
\text { overweight, myocardial infarction history }\end{array}$ & $\mathrm{R}$ & 25500 & Caudal of corona radiata, striatum and internal capsule \\
\hline 2 & 50 & M & Rheumatoid arthritis, tobacco use & $\mathrm{R}$ & 4500 & Thalamus, internal capsule \\
\hline 3 & 50 & $\mathrm{~F}$ & Hypertension & L & 4000 & Pallidum, internal capsule \\
\hline 4 & 41 & $\mathrm{~F}$ & Rheumatoid arthritis & $\mathrm{R}$ & 17000 & Thalamus, pallidum, internal capsule \\
\hline 5 & 46 & M & $\begin{array}{l}\text { Hypertension, hypercholesterolaemia, } \\
\text { overweight }\end{array}$ & $\mathrm{L}$ & 3300 & Caudate nucleus, internal capsule \\
\hline 6 & 52 & M & Hypertension, tobacco use & $\mathrm{R}$ & 10500 & Thalamus, pallidum, internal capsule \\
\hline 7 & 52 & M & Hypertension & $\mathrm{R}$ & 11600 & Thalamus, pallidum, internal capsule \\
\hline 8 & 27 & $\mathrm{~F}$ & - & $\mathrm{L}$ & 1900 & Caudate nucleus, internal capsule \\
\hline 9 & 45 & M & Hypertension & $\mathrm{L}$ & 21300 & Thalamus, pallidum, internal capsule \\
\hline 10 & 42 & $\mathrm{~F}$ & Hypertension & $\mathrm{R}$ & 18300 & Frontal lobe, caudal of corona radiate, internal capsule \\
\hline 11 & 68 & M & Hypertension, myocardial infarction history & $\mathrm{L}$ & 14000 & Thalamus, pallidum, internal capsule \\
\hline 12 & 57 & $\mathrm{~F}$ & Hypertension & $\mathrm{R}$ & 5300 & Thalamus, pallidum, internal capsule \\
\hline
\end{tabular}

FLAIR, fluid attenuated inversion recovery; $L$, left; $R$, right.

Mean age of the patients and controls did not differ: patients 48 (8.88) years (range 27-68) vs controls 48 (8.89) years (range 26-67).

Controls were recruited specifically to match the age and sex of the patients.

Barthel Index (BI). The volume of the initial lesion was estimated on magnetic resonance FLAIR images at the end of the third month (volume $=\pi / 6 \times$ length $\times$ width $\times$ the number of slice involved). Twelve age and sex matched healthy volunteers underwent DTI using the same MRI protocol.

The research protocol was approved by the local ethics committee for clinical research and all procedures involving the participant were conducted according to institutional guidelines in compliance with the regulations. Oral and written informed consent was obtained from all participants.

\section{Magnetic resonance imaging}

MRI was performed using a $1.5 \mathrm{~T}$ MRI system (Signa General Electric Medical Systems, Milwaukee, USA) equipped with gradient hardware allowing up to $23 \mathrm{mT} / \mathrm{m}$ gradients. The baseline scan was in the anteroposterior plane, using a standard head coil. Prior to scanning, head movement was limited by vacuum fixation cushions. Axial Tl weighted FLAIR, fast spin echo T2 weighted imaging, traditional FLAIR and DTI were used. Consecutive slices were acquired in an identical location for all sequences, with a $5 \mathrm{~mm}$ slice thickness. Typical acquisition parameters were: Tl-FLAIR (TR $2250 \mathrm{~ms} / \mathrm{TE}$ $7.7 \mathrm{~ms}$ ), fast spin echo T2 (TR 3800/TE120 ms) and traditional FLAIR (TR 9000/TE 120/TI 2200 ms). For DTI, an echo planar imaging sequence was used (TR10000/TE115 ms, NEX $=2$, matrix $128 \times 128$, field of view $24 \times 24 \mathrm{~cm}$ ). Diffusion weighted images were obtained with $\mathrm{b}=1000 \mathrm{~s} / \mathrm{mm}^{2}$ and diffusion sensitive gradients were applied along 13 gradient directions. In addition, a reference image without diffusion weighting $\left(b=0 \mathrm{~s} / \mathrm{mm}^{2}\right)$ was acquired. Acquisitions were repeated 20 times, and the results were averaged. The maximum strength of the diffusion gradients was $23 \mathrm{MT} / \mathrm{m}$.

\section{Image post-processing}

To correct for distortions related to eddy currents associated with the large diffusion sensitive gradients, we applied an

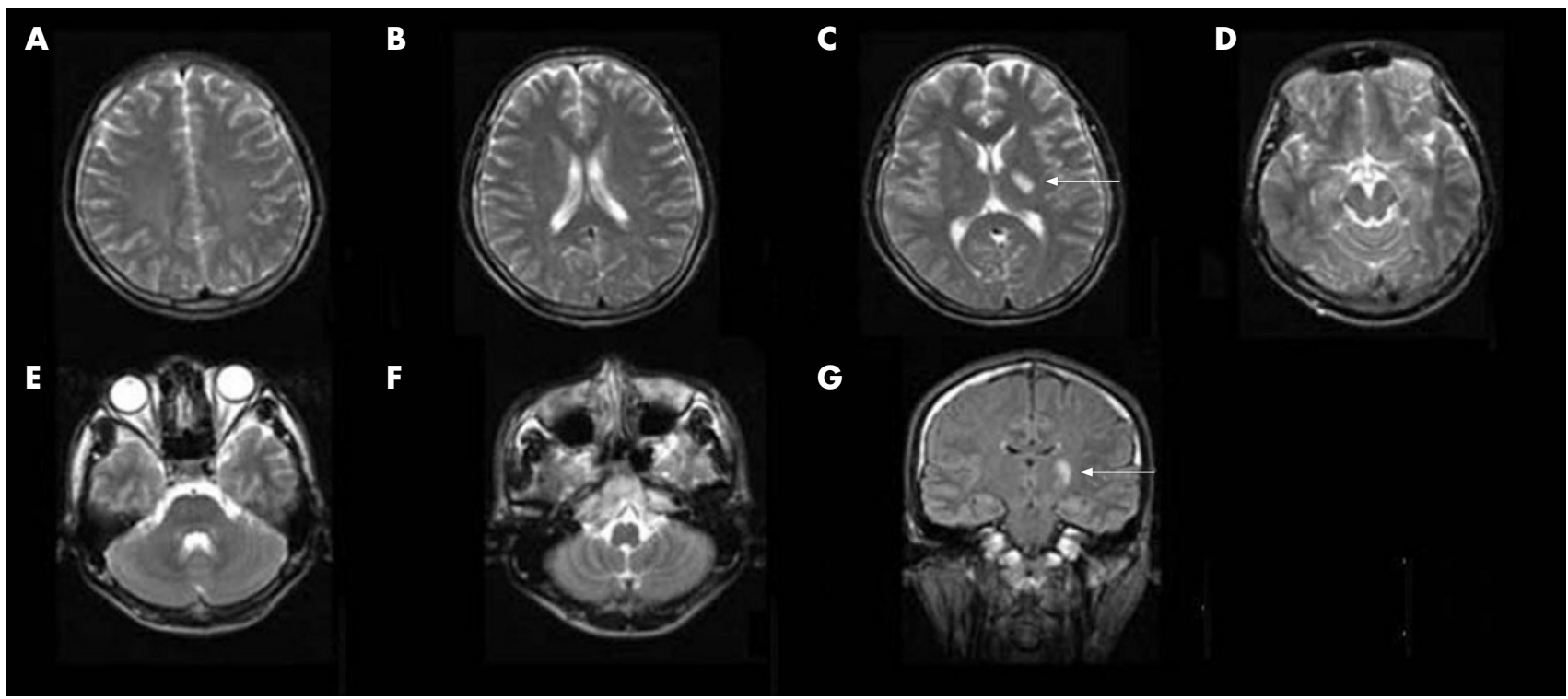

Figure 1 An example of magnetic resonance images obtained from a patient with a left subcortical infarct on the first week after the onset of stroke. From axial T2 weighted images (A-F) and the coronal fluid attenuated inversion recovery image (G), the subcortical infarct involving the posterior limb of the internal capsule is observed (white arrows). Beyond the primary lesion, there is no hyperintense signal. 


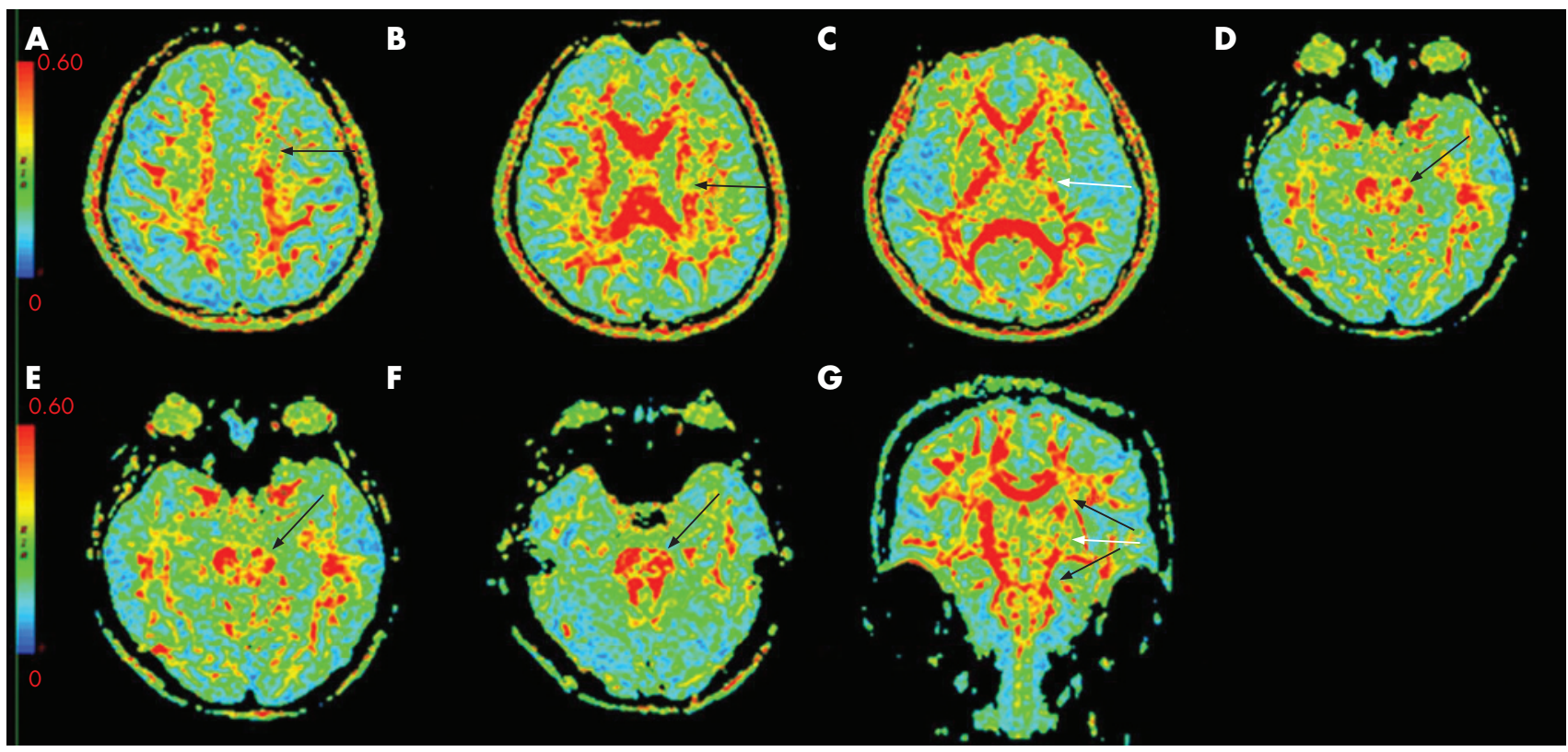

Figure 2 An example of diffusion tensor images obtained from the same patient as in fig 1 . From the axial fractional anisotropy (FA) images (A-F), reduced FA signals are observed not only at the primary lesion (C, white arrow) but in the fibre tract above the primary lesion including the centrum semiovale and coronal radiata (A, B, black arrows), and below the primary lesion including the cerebral peduncle, pons and medulla ipsilaterally (D-F, black arrows). In (G), a coronal view shows the reduced FA signals at the infarct region (white arrow) and in the fibre tract proximal and distal to the infarct lesion (black arrows).

unwrapping algorithm to the diffusion weighted data set before the tensor estimation was performed. With this correction, the diffusion tensor parameters were calculated on a pixel-by-pixel basis. With the software Fuctool 2.3.1 (Signa General Electric Medical Systems), regions of interest (ROIs) were manually defined as an ellipse with an area of $36 \mathrm{~mm}^{2}$, first on T2 weighted images and then on the corresponding DTI images. ROIs were symmetrically placed on axial slices in the left and right centrum semiovale (at the mid-point of the anterior twothirds part on the second slice over the bottom of the lateral ventricle); in the coronal radiata (at the mid-point of the anterior two-thirds part on the second slice below the bottom of the lateral ventricle); in the middle part of the posterior limb of the internal capsule along its longitudinal direction; in the anterior part of the cerebral peduncle; and in the pons and medulla. All ROI positions were determined according to the scanning baseline and the anatomical structure. The ROIs in the fibre tract both above and below the internal capsule had no abnormal signal on $\mathrm{T} 2$ weighted or FLAIR images, and therefore all were not involved with the ischaemic damage. The mean diffusivity (MD) (derived from the trace of the diffusion tensor $(\mathrm{MD}=$ trace $[\mathrm{D}] / 3)$ and measured on $\mathrm{ADC}$ images) and the fractional anisotropy index (FA value) (measured on the anisotropy images) were analysed.

\section{Statistical analysis}

Data are presented as median and quartile range. A MannWhitney test was used first for comparison between the left and right sides for median MD and FA values of any of the regions studied in controls. As the infarction appeared in either the left or right side in patients, we used median MD and FA values from both sides of the control subjects to compare with the quantitative DTI data of the patients from either the infarct side or the contralateral side to avoid any possible bias. A MannWhitney test was used for each comparison. To reveal the time progressive effect, liner regression for each patient was first established to describe the trend in clinical scores and DTI quantitative data obtained at W1, W4 and W12. The medians of within subject regression coefficients of all patients were then tested using Wilcoxon's signed ranks test to determine whether the population within subject regression coefficient was equal to zero. Finally, Spearman correlation analysis was used to assess the association between the absolute value of per cent change $(((\mathrm{Wl} 2-\mathrm{Wl}) / \mathrm{Wl}) \times 100 \%)$ of FA values and clinical scores, and the correlation between the within subject regression coefficient of the FA values and clinical scores. Values of $\mathrm{p}<0.05$ were considered statistically significant. Statistical analysis was performed using SPSS 13.0 (Abacus Concepts Inc., Chicago, Illinois, USA).

\section{RESULTS}

\section{Subject characteristics}

Six patients had only one vascular risk factor, and one patient had no known risk factors (table 1). In all patients, on T2 weighted and FLAIR images, the primary subcortical lesion involved the posterior limb of the internal capsule, and the volume of the initial lesion was obtained. All patients and controls were right handed.

\section{DTI data}

Twelve patients and controls completed all MRI examinations. Within the first week of onset, T2 weighted and FLAIR images showed hyperintensity consistent with cerebral infarction in every patient but there was no hyperintensity elsewhere (fig lAG). On DTI images, the infarct region in each patient was identified as an area of reduced FA and increased MD signal. On axial FA images (fig $2 \mathrm{~A}-\mathrm{F}$ ), the reduced signal was obvious in the primary lesion, as well as in the fibre tract proximal to the primary lesion on the infarct side (at the level of the corona radiata and centrum semiovale) and distal to the primary lesion (at the level of the cerebral peduncle, pons and medulla on the infarct side). On coronal images (fig 2G), reduced FA signal was observed in the infarct region, and spreading in opposite directions from the infarct, up along the proximal fibre 
Table 2 Fractional anisotropy value (dimensionless units) in the infarct region and at different levels of the fibre tract in patients and controls

\begin{tabular}{|c|c|c|c|c|c|}
\hline \multirow[b]{2}{*}{ Region } & \multicolumn{4}{|c|}{ FA value of patient group $(n=12)$} & \multirow{2}{*}{$\begin{array}{l}\text { FA value of control } \\
\text { group }(n=12)\end{array}$} \\
\hline & & W1 & W4 & W12 & \\
\hline \multirow[t]{2}{*}{ Centrum semiovale } & Infarct side & $0.33(0.32,0.34)$ & $0.33(0.32,0.33)$ & $0.29(0.27,0.30)$ & $0.33(0.33,0.34)$ \\
\hline & Unaffected side & $0.34(0.33,0.34)$ & $0.33(0.32,0.34)$ & $0.32(0.31,0.33)$ & $0.33(0.33,0.34)$ \\
\hline \multirow[t]{2}{*}{ Corona radiata } & Infarct side & $0.39(0.38,0.40)$ & $0.37(0.36,0.38)$ & $0.34(.32,0.35)$ & $0.40(0.39,0.41)$ \\
\hline & Unaffected side & $0.39(0.38,0.42)$ & $0.41(0.39,0.41)$ & $0.41(0.39,0.41)$ & $0.40(0.39,0.41)$ \\
\hline \multirow[t]{2}{*}{ Above the internal capsule } & Infarct side & $0.36(0.35,0.37)^{* *}$ & $0.35(0.34,0.36)^{* *}$ & $0.31(0.30,0.33)^{* *}$ & $0.37(0.36,0.38)$ \\
\hline & Unaffected side & $0.38(0.36,0.39)$ & $0.38(0.36,0.39)$ & $0.37(0.36,0.38)$ & $0.37(0.36,0.38)$ \\
\hline \multirow[t]{2}{*}{ Internal capsule } & Infarct side & $0.36(0.32,0.41)^{* *}$ & $0.35(0.24,0.40)^{* *}$ & $0.24(0.17,0.31)^{* *}$ & $0.52(0.51,0.53)$ \\
\hline & Unaffected side & $0.51(0.50,0.53)$ & $0.52(0.51,0.53)$ & $0.52(0.50,0.53)$ & $0.52(0.51,0.53)$ \\
\hline \multirow[t]{2}{*}{ Cerebral peduncle } & Infarct side & $0.44(0.43,0.45)$ & $0.42(0.41,0.43)$ & $0.39(0.37,0.40)$ & $0.47(0.47,0.48)$ \\
\hline & Unaffected side & $0.47(0.47,0.48)$ & $0.47(0.46,0.48)$ & $0.46(0.46,0.48)$ & $0.47(0.46,0.48)$ \\
\hline \multirow[t]{2}{*}{ Pons } & Infarct side & $0.40(0.39,0.41)$ & $0.38(0.38,0.39)$ & $0.36(0.34,0.37)$ & $0.42(0.41,0.43)$ \\
\hline & Unaffected side & $0.42(0.42,0.43)$ & $0.42(0.41,0.43)$ & $0.41(0.39,0.43)$ & $0.42(0.41,0.43)$ \\
\hline \multirow[t]{2}{*}{ Medulla } & Infarct side & $0.39(0.38,0.39)$ & $0.38(0.37,0.38)$ & $0.36(0.35,0.37)$ & $0.40(0.39,0.41)$ \\
\hline & Unaffected side & $0.40(0.39,0.43)$ & $0.40(0.39,0.41)$ & $0.40(0.39,0.41)$ & $0.40(0.39,0.41)$ \\
\hline \multirow[t]{2}{*}{ Below the internal capsule } & Infarct side & $0.41(0.41,0.42)^{* *}$ & $0.39(0.39,0.40)^{* *}$ & $0.37(0.36,0.39)^{* *}$ & $0.43(0.42,0.44)$ \\
\hline & Unaffected side & $0.43(0.42,0.44)$ & $0.42(0.42,0.44)$ & $0.43(0.42,0.44)$ & $0.43(0.42,0.44)$ \\
\hline
\end{tabular}

Above the internal capsule, including the corona radiata and centrum semiovale; Below the internal capsule, including the cerebral peduncle, pons and medulla; FA fractional anisotropy; W1, within the first week; W4, end of the fourth week; W12, end of the 12th week.

**Significantly different from controls, $\mathrm{p}<0.01$ (Mann-Whitney test).

Values are median $\left(Q_{L}-Q_{U}\right)$

pathway to the corona radiata and centrum semiovale, and down along the distal fibre tract to the cerebral peduncle, pons and medulla. In controls and on matched regions located on the contralateral side in patients, no obvious changes were found. On ADC images, no abnormal signal was observed outside of the primary lesion.

Compared with matched regions in controls, FA values in patients were significantly lower in the infarct and in the ipsilateral proximal and distal fibre pathway (table 2). The Wilcoxon signed ranks test showed that the population medians of the within subject regression coefficients of FA in the internal capsule $(0.011(-0.013,-0.010) ; \mathrm{p}<0.001)$, and in the fibre tract above $(-0.004(-0.004,-0.004) ; \mathrm{p}<0.001)$ and below $(-0.004 \quad(-0.004,-0.003) ; \mathrm{p}<0.001)$ the internal capsule were not equal to zero, which indicated that the FA value from these regions decreased progressively with time.

In patients, over the time period from Wl to W12, the FA value of the fibre tract above the internal capsule (derived from the mean value of the corona radiata and centrum semiovale) was reduced by $14 \%(0.36(0.35,0.37) \mathrm{Wl}$ vs $0.31(0.30,0.33)$ W12; $<<0.01)$. Similarly, in patients, the FA value of the fibre tract below the internal capsule (derived from the mean value of the cerebral peduncle, pons, and medulla) was reduced by $10 \%$ from Wl to Wl2 $(0.41(0.41,0.42)$ Wl vs $0.37(0.36,0.39)$ W12; $<<0.01)$. The FA value of every region located on the contralateral fibre tract of the patient was not significantly different from controls at any time point.

Compared with controls, MD in the infarct was decreased at Wl $\left(0.86(0.85,0.87) \mathrm{mm}^{2} / \mathrm{s}\right.$ in patients vs $1.04(1.02,1.05)$ $\mathrm{mm}^{2} / \mathrm{s}$ in controls; $\left.\mathrm{p}<0.01\right)$ but it was increased at W4 $(1.24$ $(1.20,1.26) \mathrm{mm}^{2} / \mathrm{s}$ in patients vs $1.04(1.02,1.05) \mathrm{mm}^{2} / \mathrm{s}$ in controls; $\mathrm{p}<0.01)$ and at W12 $\left(1.82(1.72,1.91) \mathrm{mm}^{2} / \mathrm{s}\right.$ in patients vs $1.04(1.02,1.05) \mathrm{mm}^{2} / \mathrm{s}$ in controls; $\left.\mathrm{p}<0.01\right)$. However, from Wl to W12, there was no significant change in MD in any region beyond the infarct area, either on the infarct side or on the contralateral side, compared with matched regions in controls.

\section{Clinical scores}

All patients had some degree of motor deficits. The FM scale ranged from 4 to $79(21.00(11.50,61.50))$ in the first examination; 11 patients had hemiplegia and one had a minor upper limb motor deficit and Broca's aphasia. Four patients had one or two limb sensory disturbances. All patients received similar therapy for stroke, and each vascular risk factor was treated appropriately. By the end of the third month, all patients recovered somewhat; 8 patients returned to work, 3 were partially dependent and 1 was completely dependent. Table 3 shows the median clinical scores at the three time points. The Wilcoxon signed ranks test showed that the population medians of the within subject regression coefficients of the clinical scores were not equal to zero. That means the NIHSS decreased but the FM scale and BI increased progressively with time.

Correlations between DTI parameters and clinical scores The per cent changes in FA values from the fibre tract above and below the internal capsule were negatively correlated with per cent changes in the NIHSS and FM scale but were not significantly correlated with BI. There was no significant correlation between the per cent changes in FA values in the internal capsule and clinical scores (table 4 ). There was only a

Table 3 Clinical scores of patients at different time points and within subject regression coefficient

\begin{tabular}{lllccc}
\hline & $\mathbf{n}$ & W1 & W4 & W12 & Regression coefficient \\
\hline NIHSS & 12 & $11.00(5.75,14.75)$ & $7.00(2.25,9.00)$ & $2.50(0.00,6.50)$ & $-0.64(-0.79,-0.31)^{* *}$ \\
FM scale & 12 & $21.00(11.50,61.50)$ & $54.00(36.25,88.50)$ & $77.00(53.25,97.75)$ & $3.66(2.61,4.34)^{* *}$ \\
BI & 12 & $40.00(25.00,82.50)$ & $57.50(46.25,100.00)$ & $95.00(61.25,100.00)$ & $3.09(1.61,4.08)^{* *}$ \\
\hline
\end{tabular}

BI, Barthel Index; FM scale, Fugl-Meyer scale; NIHSS, National Institutes of Health Stroke Scale; W1, within the first week; W4, end of the fourth week; W12, end of the 12 th week.

${ }^{* *}$ Wilcoxon's signed rank test, $\mathrm{p}<0.01$

Values are median $\left(Q_{\mathrm{L}}-Q_{U}\right)$ 
Table 4 Spearman correlation between the per cent changes in fractional anisotropy and clinical scores $(n=12)$

\begin{tabular}{|c|c|c|c|}
\hline $\begin{array}{l}\text { Region of per cent reduction } \\
\text { in } \mathrm{FA}\end{array}$ & $\begin{array}{l}\text { Per cent reduction } \\
\text { in NIHSS } r_{s}(p)\end{array}$ & $\begin{array}{l}\text { Per cent reduction } \\
\text { in FM scale } r_{s}(p)\end{array}$ & $\begin{array}{l}\text { Per cent reduction } \\
\text { in } B \mid r_{s}(p)\end{array}$ \\
\hline $\begin{array}{l}\text { Above the internal capsule } \\
\text { Internal capsule } \\
\text { Below the internal capsule }\end{array}$ & $\begin{array}{l}-0.47(0.04) \\
-0.41(0.08) \\
-0.51(0.03)\end{array}$ & $\begin{array}{l}-0.56(0.02) \\
-0.44(0.06) \\
-0.68(0.01)\end{array}$ & $\begin{array}{l}-0.39(0.09) \\
-0.11(0.27) \\
-0.22(0.19)\end{array}$ \\
\hline
\end{tabular}

significant correlation between the within subject regression coefficient of the FA value from the fibre tract below the internal capsule and NIHSS (table 5).

\section{DISCUSSION}

In the present study, all 12 patients with or without primary lesions in the basal ganglia or thalamus had infarct lesions in the internal capsule. The FA value decreased progressively from W1 to W12 in the internal capsule where the primary infarct lesion was located and on the fibre tract above and below the internal capsule on the infarct side where the primary infarct lesion was not located; MD decreased at Wl and then increased from W4 to W12 in the internal capsule but remained unchanged from Wl to W12 on the fibre tract above and below the internal capsule. The different pattern of diffusion changes in the different parts of the fibre tract indicates that the damage in the fibre tract above and below the internal capsule differs from the primary ischaemic infarct in the internal capsule. The damage in the fibre tract above and below the internal capsule may result from secondary degeneration as the pattern of diffusion changes beyond the internal capsule is similar to the WD in the peripheral nerve in experimental animal models. ${ }^{15}$

Some cross sectional studies have shown that at early or chronic stages, there is a reduction in the FA value with the MD remaining unchanged on the infarct side in the fibre tract distal to the infarct foci. In a small group of patients with a middle cerebral artery territory ischaemic stroke, 2-16 days after onset, the FA value was reduced but MD was unchanged at the cerebral peduncle on the infarct side. ${ }^{13}$ Werring et al ${ }^{9}$ reported a $15 \%$ decrease in the FA value and an unchanged MD in the fibre tract distal to the primary lesion in patients 2-6 months after a stroke. Another study found a $32 \%$ decrease in the FA value with MD unchanged in the fibre tract distal to the cerebral infarction in patients more than 1 year after a stroke. ${ }^{16}$ Only a longitudinal DTI study involving two patients with striatocapsular infarction showed that the FA value of the cerebral peduncle decreased from $16 \%$ to $25 \%$ over more than 9 months in one case, and from $17 \%$ to $48 \%$ over 3 months in another case, with a slight rise in $\mathrm{MD} .{ }^{12}$ In this study, we prospectively observed secondary degeneration in the fibre tract distal to the primary infarction in 12 stroke patient from Wl to W12 and in controls, and confirmed that the FA value decreased progressively with $\mathrm{MD}$ unchanged during the observation time period. Our data illustrate the course of secondary structural degradation more clearly, which defined WD over time in the fibre tract distal to the primary lesions. ${ }^{17} 18$

In the present study, we also found that the FA value decreased progressively with MD unchanged from W1 to W12 in the fibre tract proximal to the primary infarct. Several studies have demonstrated retrograde degeneration in the corticospinal tract after damage, and such a mechanism of "dying back" might be responsible in part. ${ }^{19-22}$ An increase in MD without a change in FA value after middle cerebral artery territory infarct was observed with DTI in the ipsilateral thalamus 1 month after stroke onset. ${ }^{23}$ Because the thalamus was remote from the primary lesion and the change in MD in the thalamus was later than in the infarct foci, it was regarded as retrograde degeneration of the thalamocortical neurons secondary to fibre damage. A case of pontine infarction with retrograde degeneration of the pyramidal tract has been detected by T2 weighted MRI and confirmed post mortem. ${ }^{14}$ It is possible that the retrograde degeneration may also occur in the coronal radiata and centrum semiovale and may be detected by DTI. In our study, the progressive reduction in the FA value in the fibre tract above the initial infarction reflects the gradual degeneration of the fibre tract proximal to the primary lesion. There are at least two fibre types in the fibre tract above the internal capsule. One is from neurons in the motor cortex, descending from the subcortex to the spinal cord and formulating the pyramidal tract. The other is derived from the relay nuclei in the thalamus that deliver somatosensory messages through the corona radiata and the centrum semiovale to sensory areas located in the frontoparietal cortex, known as the thalamic radiation. Therefore, in the fibre tract above the internal capsule, secondary degeneration in the pyramidal tract is retrograde, while in the thalamic radiation it is anterograde.

The relation between the reduction in the FA value in the fibre tract beyond the primary lesions and patient outcome is not well understood. A longitudinal study found that progressive reductions in the FA value in the pyramidal tract distal to the primary lesion were associated with persistent moderate-tosevere hemiparesis. ${ }^{12}$ Another study reported that patients with a larger degree of FA reduction at the cerebral peduncle distal to the initial infarct had a greater motor deficit in the early stage and a worse motor outcome 3 months later. In the present

Table 5 Spearman correlation between the within subject regression coefficient of fractional anisotropy value and clinical scores $(n=12)$

\begin{tabular}{lcll}
\hline Region of FA value & NIHSS $\mathbf{r}_{\mathbf{s}}(\mathbf{p})$ & FM scale $\mathbf{r}_{\mathbf{s}}(\mathbf{p})$ & BI $\mathbf{r}_{\mathbf{s}}(\mathbf{p})$ \\
\hline Above the internal capsule & $0.25(0.44)$ & $-0.38(0.22)$ & $-0.07(0.82)$ \\
Internal capsule & $-0.08(0.81)$ & $-0.126(0.70)$ & $-0.07(0.82)$ \\
Below the internal capsule & $0.86(0.000)$ & $-0.56(0.06)$ & $-0.38(0.23)$ \\
\hline
\end{tabular}

BI, Barthel Index; FA, fractional anisotropy; FM scale, Fugl-Meyer scale; NIHSS, National Institutes of Health Stroke Scale. 
study, we found that all of the stroke patients recovered gradually over 3 months, and the per cent decreases in the FA value in the fibre tract above and below the internal capsule correlated negatively with the per cent changes in NIHSS and FM scale. In addition, a significant correlation between the within subject regression coefficient of the FA value from the fibre tract below the internal capsule and NIHSS was found. These results demonstrate that during the course of neurological recovery, secondary degeneration in the fibre tract both above and below the primary lesion persists and deteriorates, and a greater extent of secondary degeneration is associated with a smaller degree of recovery. It is obvious that the secondary degeneration in the fibre tract distal and proximal to the infarction may hamper neurological recovery.

In conclusion, our study shows that secondary degeneration occurs and deteriorates not only in the fibre tract distal to but also proximal to a subcortical cerebral infarct at least 12 weeks after stroke onset, and may hamper neurological recovery. To understand the mechanism and extent of the impact of secondary degeneration on neurological recovery, future investigations with a larger sample size, more homogeneous infarcts and a longer observation period are needed.

\section{ACKNOWLEDGEMENTS}

This study was supported by grants from the Teaching and Research Award Program for Outstanding Young Teachers in Higher Education Institutions of the Ministry of Education, China (2002), the National Natural Science Foundation of China (Nos 39940012 and 30271485), the Natural Science Foundation of Guangdong Province, China (Nos 990065, 21906 and 2003C30610), China Medical Board of New York Inc. (No CMB00-730), the Fund for Priority Subjects in Clinical Medicine, Chinese Ministry of Health (2004) and the Key and Scientific Project of the Natural Science Foundation of Guangdong Province, China (Nos 2003B30303 and 2003D30301).

We thank Mr Jun Liu for statistical assistance.

\section{Authors' affiliations}

Zhiijian Liang, Jinsheng Zeng, Jian Yu, Li Ling, Department of Neurology and Stroke Centre, The First Affiliated Hospital, Sun Yat-Sen University, Guangzhou, China

Sirun Liu, Xueying Ling, Medical Imaging Centre, The First Affiliated Hospital, Jinan University, Guangzhou, China

Anding Xu, Department of Neurology, The First Affiliated Hospital, Jinan University, Guangzhou, China

The funding sources were not involved in the study design, collection, analysis or interpretation of data, writing of the report or the decision to submit the paper for publication.

Competing interests: None.

\section{REFERENCES}

1 Loos M, Dihne M, Block F. Tumor necrosis factor-alpha expression in areas of remote degeneration following middle cerebral artery occlusion of the rat. Neuroscience 2003; 122:373-80.

2 Irving EA, Bentley DL, Parsons AA. Assessment of white matter injury following prolonged focal cerebral ischaemia in the rat. Acta Neuropathol (Berl) $2001 ; 102: 627-35$

3 Dziewulska D, Rafalowska J, Podlecka A, et al. Remote morphological changes in the white matter after ischaemic stroke. Folia Neuropathol 2004;42:75-80.

4 lizuka H, Sakatani K, Young W. Corticofugal axonal degeneration in rats after middle cerebral artery occlusion. Stroke 1989:20:1396-402.

5 Dihne M, Grommes C, Lutzenburg M, et al. Different mechanisms of secondary neuronal damage in thalamic nuclei after focal cerebral ischemia in rats. Stroke 2002;33:3006-11.

6 Uchino A, Takase Y, Nomiyama K, et al. Brainstem and cerebellar changes after cerebrovascular accidents: magnetic resonance imaging. Eur Radiol 2006;16:592-7.

7 Sawlani V, Gupta RK, Singh MK, et al. MRI demonstration of Wallerian degeneration in various intracranial lesions and its clinical implications. J Neurol Sci 1997; 146:103-8.

8 Uchino A, Sawada A, Takase Y, et al. Wallerian degeneration of the middle cerebellar peduncle after pontine infarction: MR imaging. Radiat Med 2004;22:37-41.

9 Werring DJ, Toosy AT, Clark CA, et al. Diffusion tensor imaging can detect and quantify corticospinal tract degeneration after stroke. J Neurol Neurosurg Psychiatry 2000;69:269-72.

10 Le Bihan D, Mangin JF, Poupon C, et al. Diffusion tensor imaging: concepts and applications. J Magn Reson Imaging 2001;13:534-46

11 Thomalla G, Glauche V, Weiller C, et al. Time course of Wallerian degeneration after ischaemic stroke revealed by diffusion tensor imaging. J Neurol Neurosurg Psychiatry 2005;76:266-8.

12 Mukheriee P. Diffusion tensor imaging and fiber tractography in acute stroke. Neuroimaging Clin N Am. 2005;15: 655-65, xii).

13 Thomalla G, Glauche V, Koch MA, et al. Diffusion tensor imaging detects early Wallerian degeneration of the pyramidal tract after ischemic stroke. Neuroimage 2004;22:1767-74.

14 Kobayashi S, Hasegawa S, Maki T, et al. Retrograde degeneration of the corticospinal tract associated with pontine infarction. J Neurol Sci 2005;236:91-3

15 Beaulieu C, Does MD, Snyder RE, et al. Changes in water diffusion due to Wallerian degeneration in peripheral nerve. Magn Reson Med 1996;36:627-31

16 Pierpaoli C, Barnett A, Pajevic S, et al. Water diffusion changes in Wallerian degeneration and their dependence on white matter architecture. Neuroimage $2001 ; 13: 1174-85$

17 Varejao AS, Cabrita AM, Meek MF, et al. Functional and morphological assessment of a standardized rat sciatic nerve crush injury with a non-serrated clamp. J Neurotrauma 2004;21:1652-70.

18 George A, Buehl A, Sommer C. Tumor necrosis factor receptor 1 and 2 protein are differentially regulated during Wallerian degeneration of mouse sciatic nerve. Exp Neurol 2005; 192:163-6.

19 Bronson R, Gilles FH, Hall J, et al. Long term post-traumatic retrograde corticospinal degeneration in man. Hum Pathol 1978;9:602-7.

20 Kalil K, Schneider GE. Retrograde cortical and axonal changes following lesions of the pyramidal tract. Brain Res 1975;89:15-27.

21 Yamamoto T, Yamasaki M, Imai T. Retrograde pyramidal tract degeneration in a patient with cervical haematomyelia. J Neurol Neurosurg Psychiatry 1989;52:382-6

22 Pallini R, Fernandez E, Sbriccoli A. Retrograde degeneration of corticospinal axons following transection of the spinal cord in rats. A quantitative study with anterogradely transported horseradish peroxidase. J Neurosurg 1988:68:124-8.

23 Herve D, Molko N, Pappata S, et al. Longitudinal thalamic diffusion changes after middle cerebral artery infarcts. J Neurol Neurosurg Psychiatry 2005;76:200-5. 